Kruger Hendrik Gerhardus (Orcid ID: 0000-0003-0606-2053)

Baijnath Sooraj (Orcid ID: 0000-0001-7860-1779)

\title{
Mass spectrometric imaging of the brain demonstrates the regional displacement of 6- monoacetylmorphine by naloxone
}

Belin G Teklezgia , Annapurna Pamreddya ${ }^{a}$, Sphamandla Ntshangase ${ }^{a}$, Sipho Mdanda ${ }^{a}$, Sanil D. Singh $^{\mathrm{d}}$, Nirmala D Gopal ${ }^{\mathrm{b}}$, Tricia Naicker ${ }^{\mathrm{a}}$, Hendrik G Kruger ${ }^{\mathrm{a}}$, Thavendran Govender ${ }^{\mathrm{c}}$, Sooraj Baijnath $^{\mathrm{a}^{*}}$

${ }^{a}$ Catalysis and Peptide Research Unit, University of KwaZulu-Natal, Westville Campus, Durban, South Africa.

bDepartment of Criminology, University of KwaZulu-Natal, Durban, Republic of South Africa.

${ }^{c}$ Department of Chemistry, University of Zululand, Richards Bay, South Africa.

dDepartment of Pharmaceutical Science, University of KwaZulu-Natal, Westville Campus, Durban, South Africa.

\section{Correspondence to:}

*Dr. Sooraj Baijnath

Catalysis and Peptide Research Unit, E-block, 6th floor, Room E1-06-016,

University of KwaZulu-Natal, Westville Campus

South Africa, Offices: +27 31260 81799, Cell: +27 84562 1530,

Email address: baijnath.sooraj@gmail.com 


\section{Method development}

The present study was validated in compliance with European Medicine Agency (EMA) guidance for the validation of bioanalytical methods (EMA 2011). Linearity, limit of detection (LOD), lower limit of quantitation (LLOQ), extraction recovery, precision and accuracy were evaluated during method validation.

\subsection{Stock standards and quality control preparation}

Stock solutions ( $1 \mathrm{mg} / \mathrm{mL}$ ) of naloxone (NLX), 6-Monoacetylmorphine (6-MAM) and internal standard were prepared in $\mathrm{MeOH}: \mathrm{H}_{2} \mathrm{O}(50: 50)$ and stored at $-20^{\circ} \mathrm{C}$. The working solutions of NLX and 6-MAM were prepared daily to a final concentration of $10 \mu \mathrm{g} / \mathrm{mL}$ in $\mathrm{MeOH}: \mathrm{H}_{2} \mathrm{O}$, respectively. The internal standard working solution was prepared to a final concentration of $1 \mu \mathrm{g} / \mathrm{mL}$ MeOH: $\mathrm{H}_{2} \mathrm{O}(50: 50)$ and stored at $-20^{\circ} \mathrm{C}$. Calibration standards were prepared in 100 $\mu \mathrm{L}$ blank plasma or brain homogenate spiked with the working solution containing a mixture of both analytes and internal standard, providing a final concentration ranging from 0.1-50 $\mathrm{ng} / \mathrm{mL}$ and 20-1250 ng/mL for NLX and 6-MAM, respectively. Similarly, quality control (QC) samples were prepared at concentration levels of 3, 17 and $35 \mathrm{ng} / \mathrm{mL}$ for NLX and 60, 600 and $1100 \mathrm{ng} / \mathrm{mL}$ for 6-MAM representing low quality control (LQC), middle quality control (MQC) and high quality control (HQC) levels.

\subsection{Sample preparation}

Brain samples were weighed and homogenized in 3 volumes of Millipore water. An aliquots of $100 \mu \mathrm{L}$ plasma or brain homogenates were spiked with NLX or 6-MAM (to a final concentration of $200 \mathrm{ng} / \mathrm{mL}$ ) and $900 \mu \mathrm{L}$ of methanol containing internal standard solution (to a final concentration of $30 \mathrm{ng} / \mathrm{mL}$ ). After vortex-mixing (30 sec), the samples were centrifuged at $10000 \mathrm{rpm}$ for $10 \mathrm{~min}$ at $4^{\circ} \mathrm{C}$. The supernatants were collected, loaded onto a $50 \mathrm{mg}$ DSC18 SPE cartridge (SUPELCO, Pennsylvania, USA) preconditioned with $1 \mathrm{~mL}$ of $\mathrm{MeOH}$ (100\%). The samples collected were injected into LC-MS/MS analysis.

\section{Method validation}

Method validation was achieved using the following parameters; linearity, lower limit of quantification (LLOQ), limit of detection (LOD), extraction recovery, precision and accuracy, 
in compliance with the criteria set by EMA (EMA 2011). The validated method was applied for the LC-MS/MS analysis of NLX and 6-MAM in rodent biological matrices.

\section{Chromatographic conditions}

The high-performance liquid chromatography (HPLC) system was coupled with an amaZon speed ion trap (IT) fitted with an electrospray ionization (ESI) ion source from Bruker Daltonics (Bremen, Germany). The HPLC system included a Thermo Scientific Dionex UltiMate 3000 binary pump and autosampler (Dionex Softron GmbH, Germany). Chromatographic separation was conducted using a YMC $\mathrm{C}_{18}$ Triart $(150 \times 3.0 \mathrm{~mm}$ and $3 \mu \mathrm{m}$ particle size) analytical column (YMC Europe, Gmbh, Germany). The column compartment temperature was kept at $24^{\circ} \mathrm{C}$. The mobile phase consisted of Milli-Q water with $0.1 \% \mathrm{v} / \mathrm{v}$ formic acid (solvent A) and $0.1 \% \mathrm{v} / \mathrm{v}$ formic acid in methanol (solvent B). A 13 min gradient elution method was used at a flow rate of $0.3 \mathrm{~mL} / \mathrm{min}$ was accomplished as followed; $30 \%$ of mobile phase B (0-1 min), gradually increased to $90 \%$ (1-6 min), was held at $90 \%$ (6-8 min), resumed to its initial condition of 30\% (8-12min) and held at this gradient (12-13 min).

\section{Mass spectrometric analysis}

The MS was operated in the positive ionization mode with multiple reactions monitoring (MRM) for the analytes. The following MS parameters were used; capillary voltage $-4500 \mathrm{~V}$; end plate voltage $-500 \mathrm{~V}$; nebulizer -1.5 bar; dry gas temperature $-250^{\circ} \mathrm{C}$; flow-rate -8 $\mathrm{L} / \mathrm{min}$; mass range of $100-1200 \mathrm{~m} / \mathrm{z}$; ion charge control - 200000; maximum accumulation time $-200 \mathrm{~ms}$. MRM settings were as follows: isolation width was set at 2.0, 2.0, and 0.3; amplification (\%) 0.37, 0.68, 0.39, for NLX, 6-MAM and internal standard, respectively. The following precursor to product ion transitions were as follows; NLX, 328 $\rightarrow 310 \mathrm{~m} / \mathrm{z}$; 6-MAM, $328 \rightarrow 211 \mathrm{~m} / \mathrm{z}$; internal standard $342 \rightarrow 324 \mathrm{~m} / \mathrm{z}$. DataAnalysis 4.0 SP 5 and QuantAnalysis (Bruker Daltonics, Germany) were used for data acquisition and processing. 


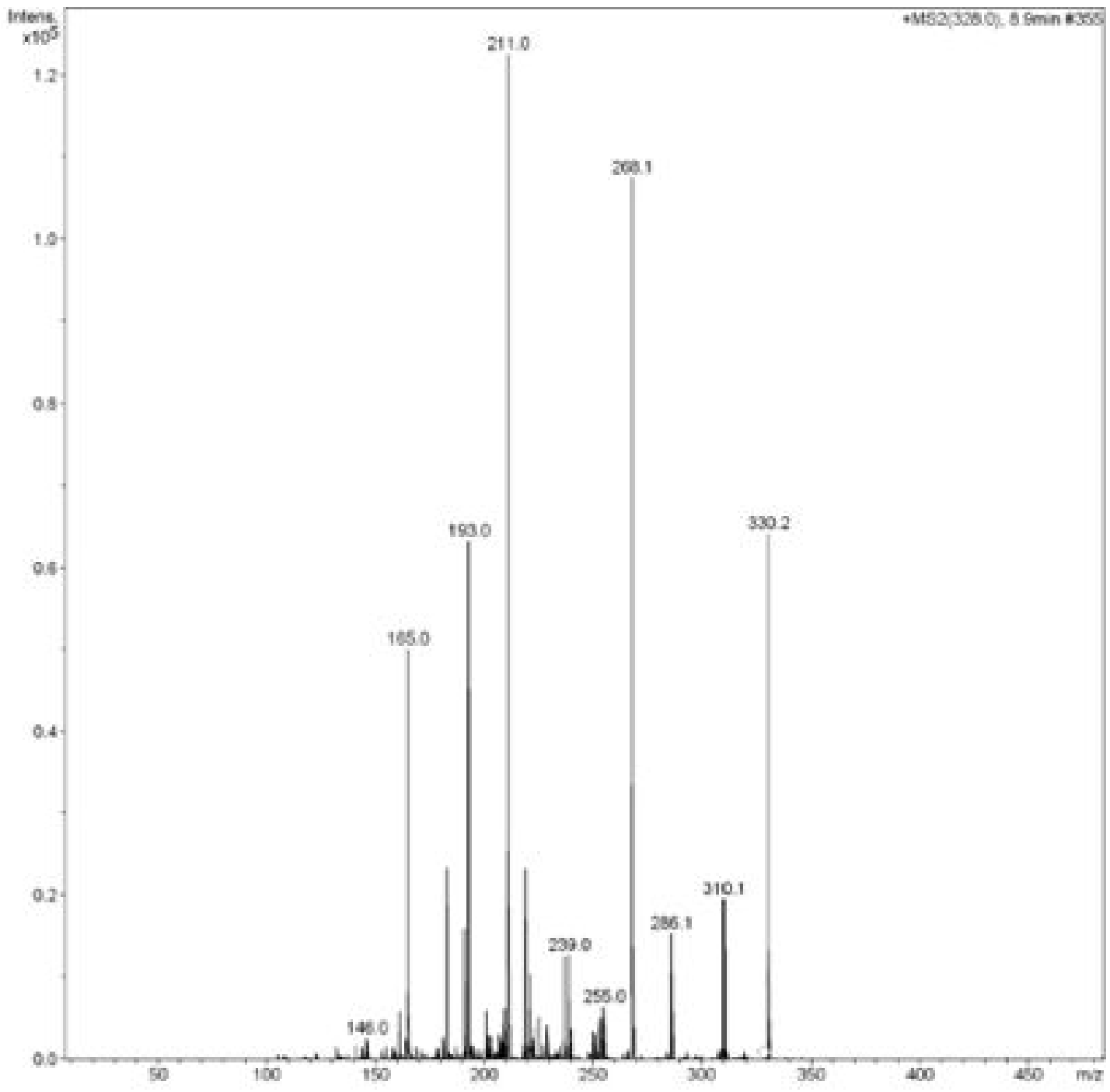

Figure S1. Representative MS/MS spectra of 6-MAM showing the analyte's fragmentation pattern. 


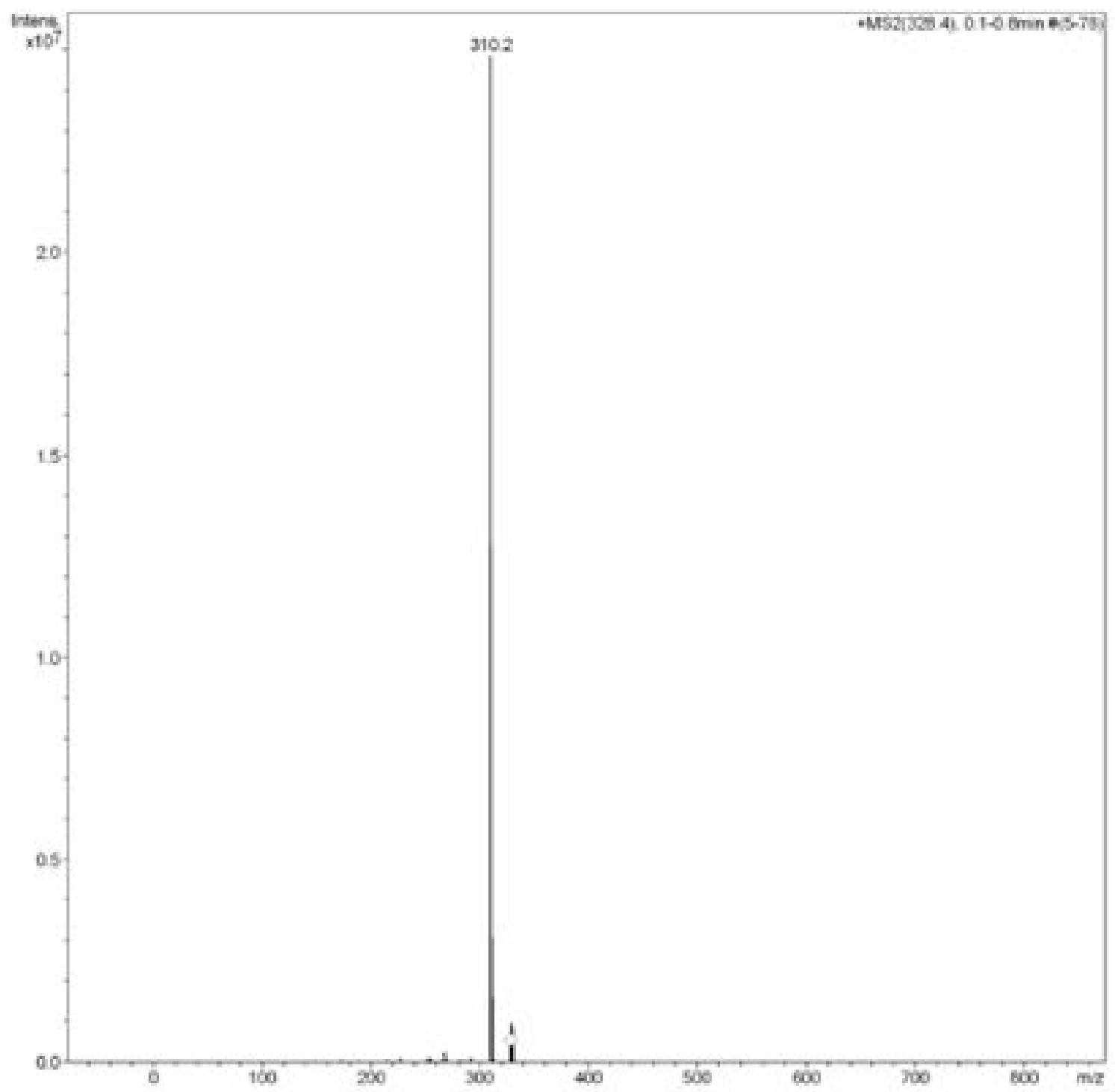

Figure S2. Representative MS/MS spectra of NLX showing the analyte's fragmentation pattern. 


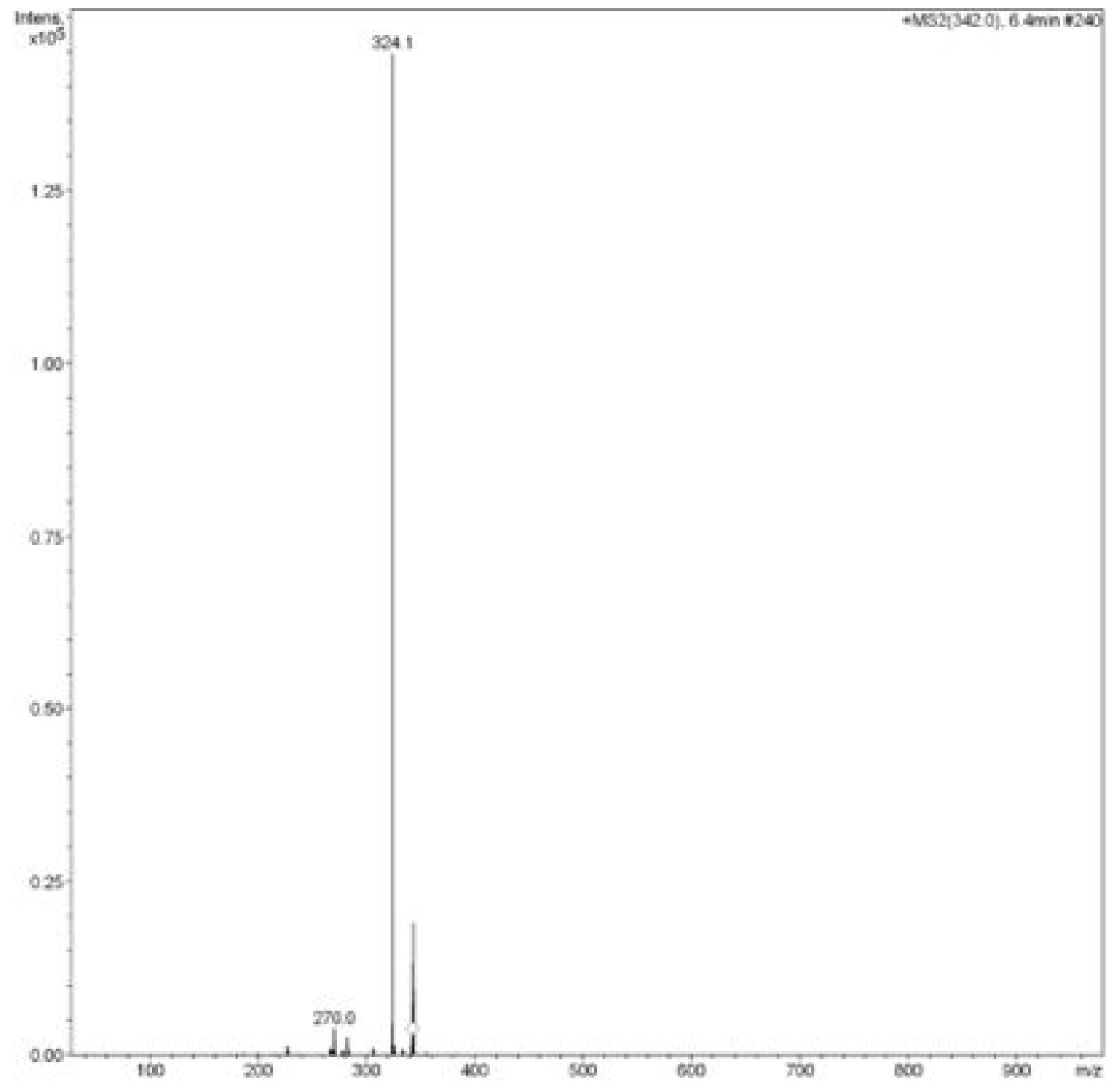

Figure S3. Representative MS/MS spectra of IS showing the analyte's fragmentation pattern. 
Table S1. Mean recoveries of NLX and 6-MAM in different biological matrices

\begin{tabular}{llcccc}
\hline \multicolumn{5}{c}{ Plasma } & \multicolumn{3}{c}{ Brain } \\
\hline Drug & Concentration & Mean Recovery (\%) & RSD (\%) & Mean Recovery (\%) & RSD (\%) \\
& level & & & \\
\hline NLX & LQC & 99.36 & 2.89 & 101.36 & 0.43 \\
& MQC & 96.96 & 3.49 & 90.96 & 6.26 \\
& HQC & 96.06 & 2.05 & 94.14 & 3.03 \\
6-MAM & LQC & 87.32 & 4.36 & 93.08 & 6.14 \\
& MQC & 87.75 & 6.51 & 90.41 & 6.78 \\
& HQC & 88.78 & 4.14 & 89.37 & 2.39 \\
\hline
\end{tabular}

$\overline{\mathrm{RSD}}=$ Relative standard deviation

Table S2. Intra and inter-day accuracy and precision for NLX in different biological matrices.

\begin{tabular}{lcccccc}
\hline & \multicolumn{3}{c}{ Plasma } & \multicolumn{3}{c}{ Brain } \\
\hline & LQC & MQC & HQC & LQC & MQC & HQC \\
\hline Theoretical concentration $(\mathrm{ng} / \mathrm{mL})$ & 3 & 7.5 & 17 & 3 & 7.5 & 17 \\
Intra-day mean concentration $(\mathrm{ng} / \mathrm{mL})$ & 2.99 & 7.49 & 16.93 & 2.88 & 7.37 & 16.77 \\
Accuracy (\%) & 99.59 & 99.97 & 99.6 & 96.0 & 98.34 & 98.68 \\
RSD (\%) & 0.433 & 6.26 & 1.13 & 2.89 & 2.72 & 4.79 \\
Inter-day mean concentration (ng/mL) & 2.97 & 7.49 & 16.98 & 2.90 & 7.41 & 16.77 \\
Accuracy (\%) & 99.26 & 99.85 & 99.92 & 96.98 & 98.77 & 98.63 \\
RSD (\%) & 1.93 & 7.49 & 6.52 & 1.57 & 2.66 & 1.47 \\
\hline
\end{tabular}

RSD $=$ Relative standard deviation 


\section{Results}

NLX and 6-MAM were simultaneously quantified using the developed LC-MS/MS method resulting in retention times (RT) of $5.8 \mathrm{~min}$ and $6.6 \mathrm{~min}$, respectively. The transitions monitored were as follows; NLX $(328 \rightarrow 310 \mathrm{~m} / \mathrm{z}), 6-$ MAM $(328 \rightarrow 268 \mathrm{~m} / \mathrm{z})$ and internal standard $(342 \rightarrow 324 \mathrm{~m} / \mathrm{z})$. The limit of detection (LOD) was determined to be $0.1 \mathrm{ng} / \mathrm{mL}$ and $4 \mathrm{ng} / \mathrm{mL}$ for NLX and 6-MAM in both brain and plasma, respectively. The lower limit of quantification (LLOQ) determined was $1 \mathrm{ng} / \mathrm{mL}$ for NLX and $20 \mathrm{ng} / \mathrm{mL}$ 6-MAM, in both matrices. NLX and 6-MAM had mean recoveries of $>85 \%$ in both matrixes, at three QC levels with RSD values of less than $15 \%$, which is within the limits set by the EMA (Table 1). Three QC levels were evaluated for Intra-day and inter-day precision and accuracy for both analytes, with the $\%$ RSD of below $15 \%$, as required by the EMA (Table $2 \& 3$ ). The LC-MS/MS method was then applied to evaluate the analytes in the biological matrices, post the administrations of the drugs in rodents.

Table S3. Intra and Inter-day accuracy and precision for 6-MAM in different biological matrices.

\begin{tabular}{lcccccc}
\hline & \multicolumn{3}{c}{ Plasma } & \multicolumn{3}{c}{ Brain } \\
\hline & LQC & MQC & HQC & LQC & MQC & HQC \\
\hline Theoretical concentration (ng/mL) & 60 & 600 & 1100 & 60 & 600 & 1100 \\
Intra-day mean concentration (ng/mL) & 55.61 & 573 & 1070 & 59.0 & 602.67 & 1096 \\
Accuracy (\%) & 94.36 & 90.59 & 90.9 & 98.5 & 100.33 & 97.33 \\
RSD (\%) & 6.5 & 11.54 & 4.94 & 6.15 & 2.29 & 2.35 \\
Inter-day mean concentration (ng/mL) & 56.04 & 562 & 1082 & 57.19 & 74 & 1094 \\
Accuracy (\%) & 93.73 & 90.37 & 90.89 & 96.5 & 96.5 & 99.5 \\
RSD (\%) & 7.27 & 11.88 & 7.33 & 6.08 & 5.09 & 1.52 \\
\hline
\end{tabular}

$\mathrm{RSD}=$ Relative standard deviation

The mean plasma concentrations of NLX and 6-MAM following the administration of a 10 $\mathrm{mg} / \mathrm{kg}$ of the drugs to the healthy SD rats are presented in Figure 1. The concentrations of 6MAM in plasma was $201.47 \mathrm{ng} / \mathrm{mL}$ after the administration of heroin $(10 \mathrm{mg} / \mathrm{kg}$ i.p) and 186.11 $\mathrm{ng} / \mathrm{mL}$ following the administration of NLX 5 min after heroin. Plasma NLX concentrations 
were $18.62 \mathrm{ng} / \mathrm{mL}$ and $15.90 \mathrm{ng} / \mathrm{mL}$ following the administration of NLX and NLX $5 \mathrm{~min}$ after heroin injection, respectively.

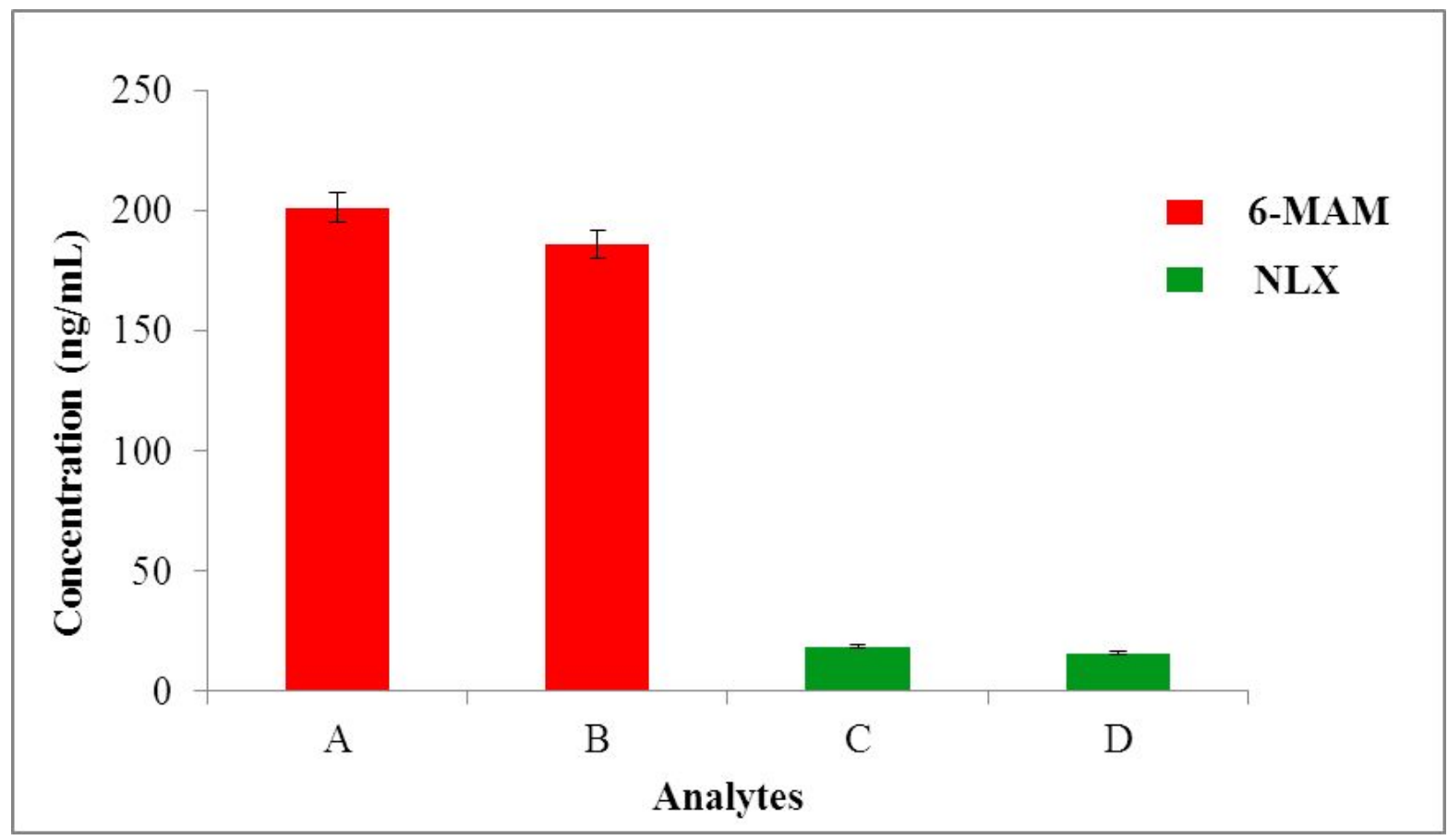

Figure S4. Quantitative data (LC-MS/MS) of 6-MAM and NLX in plasma tissues; A) 6-MAM after i.p administration of a $10 \mathrm{mg} / \mathrm{kg}$ dose of heroin, B) 6-MAM after i.p administration of a $10 \mathrm{mg} / \mathrm{kg}$ dose of heroin followed $5 \mathrm{~min}$ later by i.n administration of NLX $(10 \mathrm{mg} / \mathrm{kg}), \mathbf{C})$ NLX after i.n administration of a $10 \mathrm{mg} / \mathrm{kg}$ dose of NLX, D) NLX after i.p administration of heroin $(10 \mathrm{mg} / \mathrm{kg})$ followed $5 \mathrm{~min}$ later by i.n administration of NLX (10 mg/kg) to SD rats, 15 min post dose. 


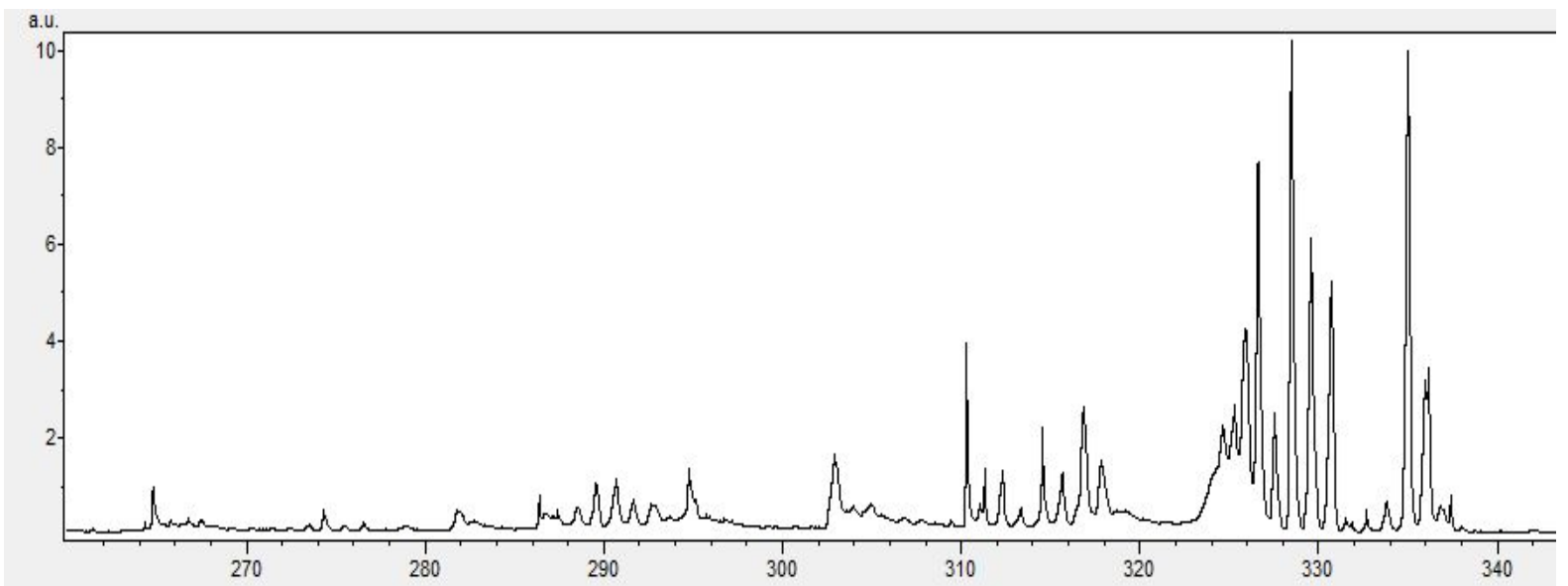

Figure S5. Representative spectra from mass spectrometry imaging experiment run on brain tissue of animals treated with heroin and NLX .

\section{References}

1. E.M.A. Guideline on bioanalytical method

validation. http://www.ema.europa.eu/docs/en GB/document library/Scientific guideline/20 11/08/WC500109686.pdf (accessed September 22). 